\title{
INFILTRAÇÃO DE ÁGUA NO SOLO EM UM LATOSSOLO VERMELHO DA REGIÃO SUDOESTE DOS CERRADOS COM DIFERENTES SISTEMAS DE USO E MANEJ $0^{(1)}$
}

\author{
J osé Antonio Maior Bono ${ }^{(2)}$, Manuel Claudio Motta Macedo(3), Cássio Antonio Tormena(4), \\ Marcos Rafael Nanni ${ }^{(4)}$, E der Pereira Gomes ${ }^{(5)} \&$ Marcelo Marques Lopes Müller $^{(6)}$
}

\begin{abstract}
RESUMO
Na região do Cerrado, as atividades agrícolas têm provocado impactos negativos, principalmente, na qualidade do solo e da água. Tanto em sistemas de exploração agrícola ou de pecuária, com di ferentes manejos, a degradação física do solo é uma das consequências da redução da produtividade. 0 objetivo deste trabalho foi verificar a infiltração de água no solo sob influência de diferentes sistemas de manejo em um Latossolo Vermelho distrófico na região dos Cerrados. Os tratamentos foram os seguintes sistemas de manejo: a) solo sob vegetação nativa; b) solo com implantação de lavoura de soja durante um ano, seguido de três anos de pastagens; c) solo com implantação de lavoura de soja durante quatro anos, segui dos por quatro anos de pastagem; d) solo com lavoura de soja contínua em sistema de plantio direto; e e) solo com pastagem continuamente. Foram avaliadas, no período de 1995 a 2002, a velocidade de infiltração básica (VIB) e a infiltração de água acumulada no solo, utilizando o método de duplos anéis concêntricos. Os usos do solo com sistema de lavoura contínua, pecuária contínua e integração lavoura-pecuária tiveram a velocidade de infiltração básica e infiltração acumulada reduzidas em relação ao solo com vegetação nativa na região do Cerrado. E ntre os sistemas estudados, o sistema
\end{abstract}

(1) Parte da Tese de Doutorado do primeiro autor apresentada ao Programa de Pós-Graduação em Solos e Nutrição de Plantas da Universidade Estadual de Maringá. Recebido para publicação em 10 de fevereiro de 2012 e aprovado em 03 de outubro de 2012.

(2) Professor Titular da Universidade Anhanguera - UNIDERP, Unidade Agrárias. Rua Alexandre Herculano, 1400, Jardim Veraneio. CEP 79037-280 Campo Grande (MS). E-mail: Ifsolo@unierp.br

(3) Pesquisador da E mbrapa Gado de Corte, BR 262 km 04. CEP 79002-970 Campo Grande (MS). E-mail: macedo@cnpgc.embrapa.br

(4) Professor Associado do Departamento de Agronomia da Universidade Estadual de Maringá - UEM. Av. Colombo, 5790. CEP 87020-900 Maringá (PR). E-mail: catormena@uem.br

(5) Professor da Universidade Federal da Grande Dourados, Faculdade de Ciências Agrárias. Caixa Postal 533. Rod. DouradosItahum, km 12. CEP 79804-970 Dourados (MS). E-mail: edergomes@ufgd.edu.br

(6) Professor Adjunto, Departamento de Agronomia, Universidade Estadual do Centro Oeste - UNICENTRO. Rua Simeão Camargo Varela de Sá, 3. Cascavel. CEP 85040-080 Guarapuava (PR). E-mail: mmuller@unicentro.br 


\title{
de integração lavoura-pecuária foi o que apresentou valores mais próxi mos aos do solo sob vegetação nativa.
}

\author{
Termos de indexação: integração lavoura-pecuária, física do solo, uso do solo e \\ manejo do solo.
}

\author{
SUMMARY: WATER INFILTRATION INTO AN OXISOL IN THE SOUTH-WEST \\ CERRADO REGION UNDER DIFFERENT USE AND \\ MANAGEMENT SYSTEMS
}

\begin{abstract}
In theCerrado region, agricultural activities havecaused negativeimpacts mainly on the soil and water quality. Both in crop and livestock systems, with different managements, the soil physical degradation has led to reduced productivity. Theobjective of this study was to investigatewater infiltration into a Dystrophic Red Latosol of theCerradoregion under different managements. The treatments consisted of the management systems: a) soil under native vegetation, b) soil with soybean for one year foll owed by three years of grazing, c) soil with implantation of soybean for four years, foll owed by four years of grazing, d) soil with continuous soybean in no-till system, e) soil with continuous grazing. From 1995 to 2002, theinfiltration rateand accumulated water infil trated in the soil wereeval uated, using the concentric ring method. In the land use systems conti nuous crop, continuous livestock and crop-livestock integration, theinfiltration rateand cumulativeinfiltration into thesoil werelower than in soil under nativeCerrado vegetation. Of thesystems studied, thevalues of theintegrated crop livestock system were closest to thesoil under nativevegetation.
\end{abstract}

Index terms: crop-livestock integration, soil physics, land use, soil management

\section{NTRODUÇÃO}

A exploração da agropecuária da região sudoeste dos Cerrados, envol vendo o sul de M ato Grosso, o sul de Goiás e o Estado de Mato Grosso do Sul, vem apresentandosérios problemas deuso emanejodosolo, causando queda na produtividade das lavouras (Kluthcouski \& Stone, 2003) edegradação das pastagens (Vilela et al., 2003), o queéatribuído a diversos fatores, entre eles a perda da qualidade física do solo. Nesse sentido, deve-se ressaltar que as práticas agrícolas adotadas sempre vêm acompanhadas de alterações do ambiente (Resende et al., 1996). Exemplo dessa alteraçãoéa reduçãona capacidadederetençãodeágua no solo, alterando o potencial matricial, a aeração do solo, a resistência do solo à penetração das raízes, a estabilidade dos agregados ea condutividadehidráulica.

O conhecimento da capacidade de infiltração de água no sol o e suas relações com as propriedades do sol osão defundamental importância para o eficiente manejo do sol o e da água (Reichardt \& Timm, 2004). Os sol os, quando cul tivados intensamenteaolongo do tempo, podem tornar-se compactados esuscetíveis à perda de sol o por erosão, como demonstrado por Lal (1984), bem como apresentar redução na sua permeabilidade (Freitas J únior \& Luchiari J únior, 1994).

Várias propriedades do solo podem interferir no processo de infiltração, entre as quais se destacam a densidadedosolo ea distribuição dotamanhodos poros
(Chen \& Wagenet, 1992). Brandão et al. (2003) sal ientam que nas condições de clima tropical e dos sol os do Cerrado brasileiro, em que predominam os óxidos de ferro e alumínio em relação às argilas silicatadas, a estrutura do sol o tem mais influência na infiltração do que a textura. E mbora os solos do Cerrado do Brasil apresentem naturalmente boas condições físicas, o manejo pode alterar as suas propriedades físicas (Vezzani, 2001; Pagotto, 2001), ocasionando redução na infil tração de água no solo. Silva \& Kato (1997) verificaram queo solo deCerrado virgem teveredução da macroporosidade de 0,39 para $0,20 \mathrm{~cm}^{3} \mathrm{~cm}^{-3}$ quando cul tivado no sistema de plantio direto; a infiltração deágua no solo passou de 26 para $1,3 \mathrm{~mm} \mathrm{~h}^{-1}$, respectivamente.

Bertol et al. (2001) verificaram redução de infiltração deágua no sol o sob pastagens compactadas pelo pisotei o animal em relação ao sol o com vegetação natural, atribuída ao aumento da densidadeeredução da macroporosidade do solo.

Sal ton et al. (1999), avaliando a infiltração deágua em solo em sistema de plantio direto sob diferentes coberturas do solo, verificaram que em sol o no qual a soja foi implantada após Brachiaria decumbens e Brachiaria brizantha foram obtidos valores superiores aos dos sol os em que as culturas de nabo forrageiro, aveia ou milho antecederam a soja. Esses autores concluem que a agregação do solo provocada pelas raízes das forrageiras influenciou positivamente a infiltração deágua no solo. 
Para Pruski et al. (1997), o sistema radicular das plantas cria caminhos quefavorecem a movimentação deágua no solo. Bono \& Macedo (1996), trabal hando com diversos sistemas de recuperação de pastagem com Brachiaria decumbens, encontraram relação positiva entre a infiltração de água no solo e a quantidade deraízes até 0,6 m de profundidade. Silva $\&$ Kato (1997) constataram aumento da infiltração de água no solo devido às coberturas vegetais, que proporcionaram aumento na macroporosidade, e redução do impacto da gota de chuva direto com a superfície do solo.

Vários model os matemáticos são utilizados para descrever o processo de infiltração de água do solo, destacando-se os de Kostiakov, Kostiakov-Lewis, Horton, Holtan, Green eAmpt e Philip (Brandão et al., 2003). Fabian \& Ottoni Filho (1997) testaram os modelos de Kostiakov, Kostiakov-Lewis, Philip e Horton em dados de infiltração coletados com infiltrômetro de anéis e concluíram que todos ajustaram adequadamente os val ores de infiltração obtidos, à exceção do modelo de Horton.

O objetivo destetrabal ho foi verificar a influência de diferentes sistemas de manejo sobrea infiltração deágua no sol o em um L atossolo Vermel ho distrófico da região dos Cerrados.

\section{MATERIAL E MÉTODOS}

A área utilizada para o experimento está localizada no Centro Nacional de Pesquisa de Gado de Corte E mbrapa Gado de Corte, em Campo Grande, Estado de Mato Grosso do Sul, nas coor denadas geográficas: latitude de $20^{\circ} 25^{\prime} 03^{\prime \prime}$ el ongitude de $54^{\circ} 42^{\prime} 20^{\prime \prime}$, cujo sol o foi descrito como Latossolo Vermel ho distrófico (Embrapa, 1997).

O local foi desmatado entre 1972 e 1973, no qual havia vegetaçãotípica de cerradão(Ferri, 1977); foram implantadas pastagens de Brachiaria decumbens, que foi utilizada para pastejo na al imentação do rebanho da E mbrapa Gado de Corte. Em outubro de 1987, foi realizada uma gradagem pesada, retirada das raízes, calagem (1,0 t ha-1 PRNT $100 \%$ ) eadubação da área com $63 \mathrm{~kg} \mathrm{ha}^{-1} \mathrm{deP}_{2} \mathrm{O}_{5}$ (superfosfatosimples), $60 \mathrm{~kg} \mathrm{ha}^{-1}$ de $\mathrm{K}_{2} \mathrm{O}$ (cloreto de potássio), $1,2 \mathrm{~kg} \mathrm{ha}^{-1}$ de $\mathrm{Zn}, 0,4$ $\mathrm{kg} \mathrm{ha}^{-1}$ de $\mathrm{B}, 0,8 \mathrm{~kg} \mathrm{ha}^{-1}$ de $\mathrm{Mn}$ e 0,4 $\mathrm{kg} \mathrm{ha}^{-1}$ de $\mathrm{Cu}$ (FTE), incorporados com gradeniveladora. No período de 1973 a 1987, o solo apresentava as seguintes características químicas (Quadro1) egranulométricas (Quadro 2) de acordo com métodos descritos em Embrapa (1997).

Novas cal agem eadubação foram efetuadas entre setembro e outubro de 1993: a calagem, para atingir uma saturação por bases entre 40 e 45 \%; em seguida, uma gradagem niveladora para uniformização da área e incorporação do calcário. Após a calagem, foi real izada uma adubação fosfatada $\left(80 \mathrm{~kg} \mathrm{ha}^{-1} \mathrm{deP}_{2} \mathrm{O}_{5}\right)$, utilizando-secomo fonteosuperfosfatosimples aplicado a lanço e incor porado com grade niveladora.

\section{Descri ção dos tratamentos}

Neste trabalho foram considerados cinco tratamentos de manejo do solo: CERR - solo com vegetação nativa; PCCA - solo com pastagem Brachiaria decumbens cultivar Basilisk contínua e adubada; L IP3 - solo com um ano sob lavoura de soja e três anos sob pastagem com Brachiaria brizantha cultivar Marandu; L1P3 - sol o com umano sob lavoura de soja e três anos sob pastagem com Brachiaria brizantha cultivar Marandu; L 4P4 - sol o com quatro anos com lavoura de soja equatro anos sob pastagem com Panicum maximum cultivar Tanzânia-1; eLCPD - lavoura contínua desoja em sistema de plantiodireto. As amostragens nos tratamentos iniciaram-sea partir de julho de 1995, após dois anos da sua implantação.

No tratamento PCCA, o sistema foi de pastagem contínua, sendo implantada a forrageira Brachiaria decumbens cv. Basilisk em parcelas com dimensões de $140 \times 50$ m (7.000 m²) e adubada a cada dois anos. As adubações utilizadas equival eram ao uso de $400 \mathrm{~kg} \mathrm{ha}^{-1}$ doformulado00-20-20, aplicadoa lanço e incorporado na camada de $0-10 \mathrm{~cm}$ na implantação e, após, somente superficial, realizada nos meses de novembro; a partir de 2000/2001, a pastagem passou a receber anualmente $50 \mathrm{~kg} \mathrm{ha}^{-1}$ de $\mathrm{N}$, tendo como fontea ureia, o que foi feito entre dezembro ejaneiro.

No tratamento LCPD, manejo com lavoura contínua, em sistema de plantio direto, em parcelas com dimensões de $110 \times 12 \mathrm{~m}\left(1.320 \mathrm{~m}^{2}\right)$, o preparo inicial foi feito como descrito anteriormente. No ano agrícola de 94/95, iniciou-se o plantio de soja como cultura de verão, não ocorrendo mais o preparo do sol o. A adubação do solo era feita na cultura de verão, utilizando-se 400 a $450 \mathrm{~kg} \mathrm{ha}^{-1}$ do formulado 00-2020. No cultivo de inverno, foram utilizados o milheto e o sorgo como culturas para formação de palhada. $\mathrm{Na}$ área, foi efetuada nova aplicação superficial de calcário noano de 1998, para manter a saturação por bases a $60 \%$, na dose de $1.800 \mathrm{~kg} \mathrm{ha}^{-1}$. A média da produtividade de soja no período estudado ficou em $2.435 \mathrm{~kg} \mathrm{ha}^{-1}$.

No tratamento L1P3, o sistema de manejo foi de um ano com lavoura desoja, seguido por três anos de pastagem. Com exceção do ano de 1993, no qual a lavoura de soja foi introduzida com o preparo convencional do solo, o plantio da soja foi realizado sem o preparo do sol o (plantio dir eto), assi m como o plantio da forrageira. A forrageira utilizada nesse sistema foi a Brachiaria brizantha cv. Marandu. Na pastagem, foi aplicada adubação anual de manutenção, com $200 \mathrm{~kg} \mathrm{ha}^{-1}$ da fórmula 0-20-20 e $80 \mathrm{~kg} \mathrm{ha}^{-1}$ de $\mathrm{N}$, utilizando ureia como fonte. Nova calagem foi realizada para manter a saturação por bases a $60 \%$ no ano de 1997, na dose de $1.500 \mathrm{~kg} \mathrm{ha}^{-1}$. A partir de 1997 o manejo do solo foi em sistema de plantio direto, tanto da lavoura como da pastagem. A 
Quadro 1. Características químicas do solo da área em estudo antes da implantação do experimento, nas camadas avaliadas

\begin{tabular}{|c|c|c|c|c|c|c|c|c|c|}
\hline Prof. ${ }^{(1)}$ & $\mathrm{pH}\left(\mathrm{H}_{2} \mathrm{O}\right)$ & $\mathbf{P}$ & K & $\mathrm{Ca}^{2+}$ & $\mathrm{Mg}^{2+}$ & $\mathrm{Al}^{3+}$ & H+AI & MO & $\mathbf{v}$ \\
\hline $\mathrm{m}$ & & \multicolumn{2}{|c|}{$-\mathrm{mg} \mathrm{dm}^{-3}$} & \multicolumn{4}{|c|}{$-\mathrm{cmol}_{\mathrm{c}} \mathrm{dm}^{-3}$} & $\mathrm{~g} \mathrm{dm}^{-3}$ & $\%$ \\
\hline $0,0-0,20$ & 5,14 & 1,5 & 43 & 0,55 & 0,27 & 0,95 & 7,24 & 32,0 & 11,4 \\
\hline $0,2-0,40$ & 5,02 & 1,0 & 25 & 0,34 & 0,19 & 1,00 & 6,55 & 21,0 & 8,2 \\
\hline
\end{tabular}

Quadro 2. Características granulométricas, argila dispersa em água (ADA) e grau de floculação (GF) do solo da área em estudo antes da implantação do experimento

\begin{tabular}{cccccc}
\hline Prof.(1) & Argila & Silte & Areia & ADA & GF \\
\hline m & & \multicolumn{3}{c}{ kg $^{-1}-$} & \\
\cline { 3 - 5 } $0,00-0,10$ & 380 & 100 & 520 & 80 & 789 \\
$0,10-0,20$ & 387 & 98 & 515 & 100 & 742 \\
$0,20-0,40$ & 410 & 120 & 470 & 110 & 732 \\
$0,40-0,60$ & 412 & 119 & 469 & 115 & 721 \\
\hline (1) & & & & &
\end{tabular}

(1) Profundidade

média da produtividade de soja no período estudado ficou em $2.520 \mathrm{~kg} \mathrm{ha}^{-1}$.

Notratamento L4P 4, as parcelas foram utilizadas com a cultura da soja por quatro anos, seguidos por quatro anos de pastagem utilizando a forrageira Panicum maximum cv. Tanzânia-1. No período de 1993 a 1996, o plantio da soja foi feitoem sol o preparado com grade; a partir de 1996, o plantio foi feito sem o preparo do solo (plantio direto). A cultura da soja recebeu adubação de $80 \mathrm{~kg} \mathrm{ha}^{-1}$ de $\mathrm{P}_{2} \mathrm{O}_{5}$ e $80 \mathrm{~kg} \mathrm{ha}^{-1}$ de $_{2} 0$, utilizando o formulado 00-20-20 para cultivo. A pastagem recebeu adubação anual de manutenção composta de $200 \mathrm{~kg} \mathrm{ha}^{-1}$ da fórmula 00-20-20 e $100 \mathrm{~kg}$ $\mathrm{ha}^{-1}$ de $\mathrm{N}$, utilizando ureia como fonte. Nova calagem foi realizada para manter a saturação por bases em $60 \%$ no ano de 1997 , na dose de $1.500 \mathrm{~kg} \mathrm{ha}^{-1}$. A média da produtividade de soja no período estudado foi de $2.705 \mathrm{~kg} \mathrm{ha}^{-1}$.

\section{Delineamento estatístico}

O delineamento estatístico foi o deblocos ao acaso distribuídos em parcelas experimentais em delineamento de blocos inteiramentecasualizados com quatro repeti ções, sendo os tratamentos considerados como parcela principal, e os anos, como parcelas subdivididas.

\section{Manejo das forragei ras}

As pastagens utilizadas foram Brachiaria decumbens cv. Basilisk, Brachiaria brizantha cv. Marandu e Panicum maximum cv.Tanzânia-1. A semeadura das gramíneas foi em 1994, no mês de outubro, a lanço, nas quantidades de 1,8, 3,0 e 2,0 $\mathrm{kg} \mathrm{ha}^{-1}$ de sementes puras viáveis, respectivamente para Brachiaria decumbens, Brachiaria brizantha e Panicum maximum.

As forrageiras foram pastejadas em sistema contínuo, com taxa de lotação variável de acordo com a disponibilidade de forragem, preestabel ecida entre 2.500 e $3.000 \mathrm{~kg} \mathrm{ha}^{-1}$ dematéria seca total, utilizando como categoria animal bezerras da raça nel ore com peso vivo entre 200 e $220 \mathrm{~kg}$ por animal. A carga animal média anual para as áreas com Brachiaria decumbens e Brachiaria brizantha, durante o período estudado, ficou em torno de 1,6 unidade animal (UA). Para as áreas com Panicum maximum, a carga média anual foi de 1,7 UA.

\section{Velocidade de infiltração básica (VIB) e infiltração acumulada de água no solo}

A infiltração deágua no solo foi determinada pelo método do infiltrômetro de duploanel. Os anéis foram cravados nosolo por percussão, de forma concêntrica; o anel interno com diâmetro de 0,3 m foi cravado no soloaté $0,10 \mathrm{~m}$, eo externo de 0,6 m de diâmetro, até $0,05 \mathrm{~m}$ (Forsytthe, 1985). Para regular a entrada de água nos anéis e manter a mesma carga hidráulica noanel interno, adaptou-seuma estrutura demadeira eboias (Figura 1).

Dentro decada parcela experimental, determinouse uma área útil de $300 \mathrm{~m}^{2}$ para realização dos testes (Figura 2). Na área útil foram demarcados quatro locais, que serviram de pontos onde se real izaram os testes de infiltração nos anos de 1995, 1996, 1997, 1998, 1999, 2000, 2001 e 2002. Os testes deinfiltração duraram, em média, 6 h e foram real izados com solo seco entre os meses de jul ho e agosto - os mais secos do ano na região de Campo Grande(MS).

Para iniciar os testes, adicionava-se água simultaneamente nos dois anéis até a altura de 0,075 m delâmina deágua. A partir dessemomento, começava-se a contagem dotempo, mantendo a carga hidráulica de 0,075 m no anel interno e 0,05 $\mathrm{m}$ no anel externo.

Os valores de infiltração foram obtidos por meio da equação 1:

Infiltração $\left(\mathrm{cm} \mathrm{h}^{-1}\right)=\frac{\text { Volume infiltrado }\left(\mathrm{cm}^{3}\right)}{\text { Área do anel interno }\left(\mathrm{cm}^{2}\right) \times \text { tempo }(h)}$ 
Aos dados ajustou-seo model omatemático proposto por Philip (Equações 2 e3), conformeForsythe(1985) e Brandão et al. (2003), para determinar a vel ocidade deinfiltração básica ea infiltração acumulada nosolo:

$$
\mathrm{VI}=\mathrm{aT}^{-0,5}+\mathrm{b}
$$

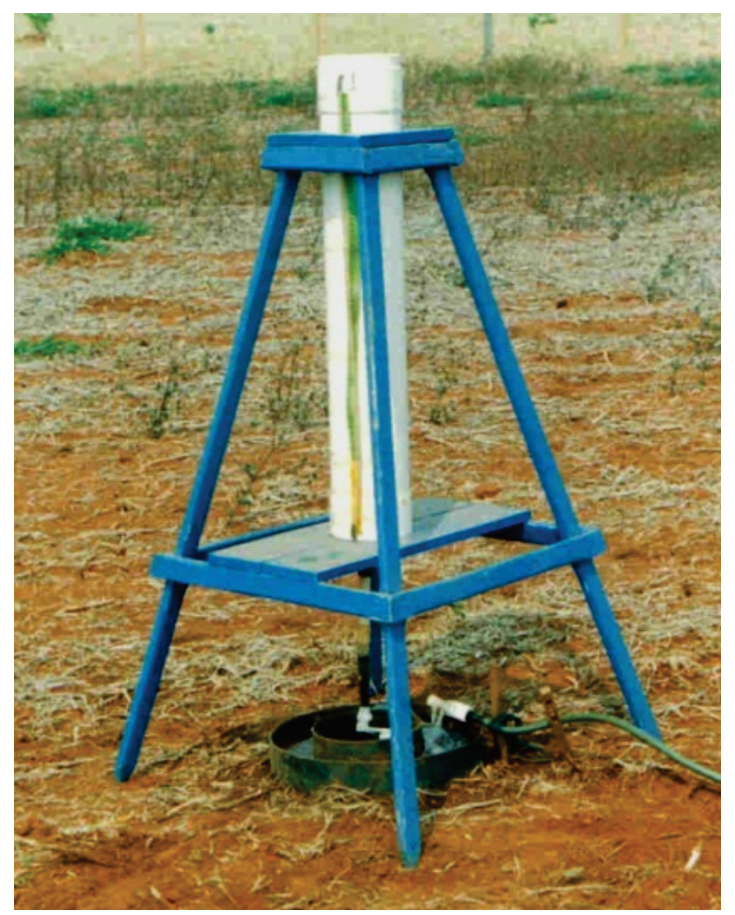

Figura 1. Detalhe dos anéis, da estrutura e das boias utilizadas na determinação da velocidade de infiltração básica (VI B) e infiltração acumulada deágua no solo.

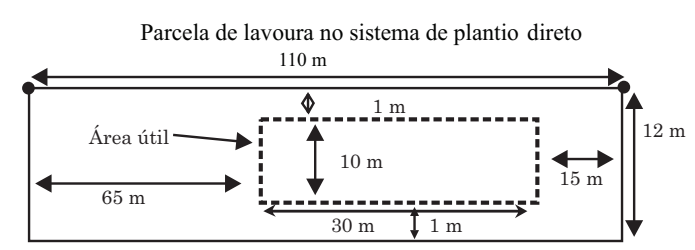

Parcela com pastagem

$140 \mathrm{~m}$

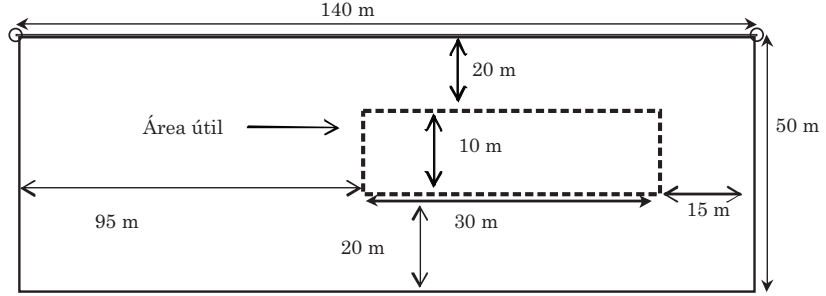

Figura 2. Esquema utilizado para avaliação da infiltração estável e da infiltração de água no solo nos tratamentos de lavoura contínua, pastagem contínua e integração lavourapecuária. em que, VI = velocidadedeinfiltração básica $\left(\mathrm{cm} \mathrm{h}^{-1}\right)$; $T$ = tempo (h); e "a" e "b" = parâmetros de ajuste do modelo.

Para determinar a infiltração acumulada (IA), os dados foram ajustados utilizandoa equação:

$$
\mathrm{IA}=\mathrm{CT} 0,5+\mathrm{d}
$$

em que, IA = infiltração acumulada (cm); T = tempo (h) e "c"e "d" = parâmetros do modelo.

\section{Porosi dade e densidade do solo}

Em dezembro de 1996, 1999, 2000, 2001 e 2002 foram col etadas amostras indeformadas, em quatro locais dentro de cada parcela, utilizando-se anéis metálicos de capacidade de $0,0001 \mathrm{~m}^{3}$, em quatro profundidades: $0-0,1 ; 0,1-0,2 ; 0,2-0,3$ e $0,3-0,4 \mathrm{~m}$. Os anéis foram introduzidos no solo por percussão, utilizando amostrador para amostra indeformada.

Nas amostras coletadas foram determinadas: a densidade de partícula do sol o (Dp), pel o método do balão vol umétrico; a densidadedosolo(Ds), pelométodo do anel vol umétrico; e o volumetotal de poros (VTP), por meio das densidades do solo e de partícula (E mbrapa, 1997). A microporosidade (Mi) foi determinada pela água retida na tensão de 0,006 MPa, utilizando o método da centrífuga adaptado por Freitas J únior \& Silva (1984). A macroporosidade (Ma) foi obtida pela diferença entre VTP e a Mi (Embrapa, 1997).

\section{E stabili dade dos agregados do solo em água}

Em dezembro de 1995, novembro de 2000 e fevereiro de 2002, foram col etadas amostras de solo em quatro locais nas camadas de 0-0,10 e 0,10$0,20 \mathrm{~m}$. As amostras foram passadas em peneira com $8 \mathrm{~mm}$ de mal ha e col etadas na peneira de $2 \mathrm{~mm}$, conforme Guedes et al. (1996), sendo posteriormente secas ao ar. E m seguida, procedeu-se ao agitamento das amostras, em peneiramento por via úmida (Kemper \& Chepil, 1965). Utilizaram-se $100 \mathrm{~g}$ de agregados secos ao ar, umedecidos por capilaridade, os quais foram colocados na parte superior de um conjunto de cinco peneiras com diâmetro de abertura da malha de 2,00; 1,00; 0,50; 0,25 e 0,105 mm, agitando-as verticalmente com 46 oscilações verticais por min dentro da água, durante $10 \mathrm{~min}$. Nas amostras dos agregados secos ao ar, determinou-sea umi dade pel o método gravimétrico (Embrapa, 1997), para corrigir a massa nos agregados submetidos ao tamisamento. Em função dos diâmetros de mal ha das penei ras utilizadas, os agregados foram distribuídos nas seguintes classes de diâmetro equival ente: $8-2$; 2-1, 1-05; 0,5- 0,25 e 0,25-0,105 e abaixo de 0,105 $\mathrm{mm}$.

A partir da massa dos agregados estáveis emágua retidos nas peneiras, determinou-seo diâmetro médio ponderado (DMP) utilizando a equação 4, conforme Kemper \& Chepil (1965). 


$$
D M P=\sum_{i}^{n}(x i w i)
$$

em que, wi = propor ção de cada classe em relação ao total; exi = diâmetro médio das classes, expresso em $\mathrm{mm}$.

\section{Análises estatísticas}

Osval ores das variáveis velocidadedeinfiltração básica e infiltração acumulada foram interpretados utilizando-se a análise de variância; para comparar as médias, aplicou-se o teste de Tukey a $5 \%$. Na infiltraçãoacumulada, realizou-seanálisedevariância para cada hora do teste.

Foi real izadoestudo decorrelação conformePimentelGomes \& Garcia (2002) entrea densidadedo solo, macro e microporosidadee diâmetro médio ponderado coma taxa devel ocidade deinfiltração básica, com os valores médios para as duas camadas desolo.

\section{RESULTADOS E DISCUSSÃO}

\section{Velocidade de infiltração bási ca (VIB)}

Os valores médios da velocidade de infiltração básica (VIB) do sol o nos diferentes sistemas demanejo encontram-se no quadro 3. A VIB apresentou interação significativa para tratamentos e ano ( $p>0,01)$. A interação foi desdobrada, analisando-seo efeito de ano dentro de cada tratamento (Quadro3).

O sol o com vegetação nativa não teve variaçãona taxa de infiltração estável de água no solo ao longo dos seteanos do estudo efoi oqueapresentou o maior valor em relação aos demais sistemas demanejo. Esses resultados confirmam os obtidos por Bertol et al.
(2001), sendo indicativo das boas condições físicas dos sol os do Cerrado sob vegetação nativa.

A remoção da vegetação natural e a introdução, seja de pastagem ou de lavoura, trazem como consequência a redução na vel ocidade de infiltração básica de água no solo, confirmando os resultados encontrados por Silva \& Kato (1998).

Na comparação entre o PCCA eo LCPD, observousequeosistema delavouras apresenta menor VIB. Stone et al. (2001) verificaram que a gramínea Brachiaria deaumbens, quando fertilizada, podeproporcionar maior estruturação do solo, aumentando sua permeabilidade. Noentanto, notratamento L 1P3, a VIB foi a mais baixa entre os tratamentos, na média dos anos da avaliação. Essa menor taxa de infiltração no L1P3 éatribuída ao tipo decrescimento cespitoso da Brachiaria brizantha, o que deixa o sol o exposto (Zimmer et al., 1994), eisso faz com que semanifeste o efeito dos cascos dos animais na superfíciedosolo, compactando-o, e, consequentemente, a taxa de infiltração. Os resultados apresentados em Bertol et al . (1997) eLanzanova et al. (2005) confirmam a influência do pisoteioanimal na redução deinfiltração deágua nosolo.

Deve-seressaltar quenosistema demanejo PCCA, devido ao sistema radicular muito agressivo dessa espécie, oque proporciona maior produção de matéria seca da parte aérea e de raízes, cobrindo bem a superfície do solo, podeter reduzido oefeito do pisoteio. A relaçãodireta entrea produção debiomassa radicular ea de biomassa verdena Brachiaria decumbens pode ser observada notrabal ho deSilva et al. (1998) eKanno et al. (1999), com esteúltimo trabal hando no mesmo experimento do presenteestudo.

No sistema de L4P4, verificou-se redução da infiltração de $46,75 \mathrm{~cm} \mathrm{~h}^{-1} \mathrm{em} 1995$ para $18,12 \mathrm{~cm} \mathrm{~h}^{-1}$ em 1998, período em que o solo foi cultivado com a cultura da soja em sistema convencional. A partir de

\section{Quadro 3. Valores médi os obtidos de velocidade de infiltração básica (VIB) no solo em diferentes sistemas de manejo do solo no Cerrado}

\begin{tabular}{|c|c|c|c|c|c|}
\hline Ano & CERR & L1P3 & L4P4 & LCPD & PCCA \\
\hline & & & $\mathrm{cm} \mathrm{h}^{-1}$ & & \\
\hline 1995 & 86,12 a & $24,75 \mathrm{a}$ & $46,75 \mathrm{a}$ & 59,62 a & $26,5 \mathrm{~d}$ \\
\hline 1996 & $85,50 \mathrm{a}$ & $16,62 \mathrm{~b}$ & $24,12 \mathrm{bc}$ & $37,00 \mathrm{~b}$ & 38,11 a \\
\hline 1997 & 93,87 a & $17,62 \mathrm{~b}$ & $19,62 \mathrm{c}$ & $21,62 \mathrm{c}$ & $29,25 \mathrm{c}$ \\
\hline 1998 & 88,00 a & $11,50 \mathrm{c}$ & $18,12 \mathrm{c}$ & $14,00 \mathrm{~d}$ & $34,37 \mathrm{ab}$ \\
\hline 1999 & 90,37 a & $11,75 \mathrm{c}$ & $27,50 \mathrm{~b}$ & $15,87 \mathrm{~d}$ & $39,50 \mathrm{a}$ \\
\hline 2001 & 92,62 a & $13,62 \mathrm{c}$ & $27,50 \mathrm{~b}$ & $14,87 \mathrm{~d}$ & $36,12 \mathrm{ab}$ \\
\hline 2002 & 85,37 a & $11,75 \mathrm{c}$ & $29,37 \mathrm{~b}$ & $11,37 \mathrm{~d}$ & $32,25 \mathrm{bc}$ \\
\hline Média & 88,83 & 15,37 & 27,56 & 24,90 & 33,72 \\
\hline
\end{tabular}

CERR: solo com vegetação natural; PCCA: solo com pastagem contínua e adubada; L1P3: solo com lavoura de soja de um ano e três anos com pastagem de Brachiaria brizantha; L4P4: solo com lavoura de quatro anos e quatro anos com pastagem de Panicum maximum; LCPD: solo com lavoura de soja em sistema de plantio direto. Valores com letras iguais na coluna não diferem entre si pelo teste de Tukey a $5 \%$. 
1998 houve a introdução da forrageira Panicum maximum cv. Tanzânia-1, e a adubação residual deixada pelo manejo da lavoura favoreceu o estabel ecimento da forrageira, proporcionandosistema radicular mais abundantee profundo, o quecontribuiu para aumentar a VIB a partir de 1999.

Kanno et al. (2001) registram que a forrageira Panicum maximum, em solo com níveis adequados de nutrientes, apresenta capacidade de produção de biomassa, tanto da parteaérea como das raízes, superior à de Brachiaria decumbens. Bono et al. (1995), utilizando forrageiras dos gêneros Brachiaria e Panicum e dois níveis de adubação e calcário, registraram aumento na condutividadehidráulica do sol o sob as doses mais al tas deadubação ede cal cário.

No quadro 4 encontram-seas correlações entrea vel oci dade de infiltração básica (VIB) e a porosidade em diferentes sistemas de manejo do solo. Verifica-se relação positiva e significativa da VIB e a macroporosidade do sol oetodos os sistemas demanejos estudados. A dependência entreas variáveis foi mais efetiva para o sistema PCCA, observada pelo maior coeficiente angular do modelo linear ajustado entrea VIB ea macroporosidade, nessetratamento.

As correlações significativas entre taxa de infiltraçãoe macroporosidade, independentementedo

Quadro 4. Correlação entre a velocidade de infiltração básica (VIB) de água e a macro e microporosidade, e entre o diâmetro médio ponderado (DMP) em diferentes sistemas de manejo do solo no Cerrado

\begin{tabular}{|c|c|c|c|}
\hline \multicolumn{2}{|c|}{ Tratamento } & \multirow{2}{*}{$\begin{array}{c}\text { Equação de correlação } \\
\hat{y}=0,0713+0,0019 x\end{array}$} & \multirow{2}{*}{$\frac{\mathbf{R}^{\mathbf{2}}}{0,56^{*}}$} \\
\hline \multirow{3}{*}{ CERR } & Macro & & \\
\hline & Micro & $\hat{\mathrm{y}}=0,2849+3 \mathrm{E}-05 \mathrm{x}$ & $0,00001^{\mathrm{ns}}$ \\
\hline & DMP & $\hat{y}=2,066+0,008 x$ & $0,18^{\mathrm{ns}}$ \\
\hline \multirow{3}{*}{ PCCA } & Macro & $\hat{y}=-0,0647+0,0074 x$ & $0,77^{* *}$ \\
\hline & Micro & $\hat{y}=0,3382+0,0006 x$ & $0,042^{\text {ns }}$ \\
\hline & DMP & $\hat{y}=-0,387+0,0983 x$ & $0,65^{* *}$ \\
\hline \multirow{3}{*}{ L1P3 } & Macro & $\hat{y}=0,0829+0,0037 x$ & $0,51^{*}$ \\
\hline & Micro & $\hat{y}=0,3388+0,0006 x$ & $0,031^{\mathrm{ns}}$ \\
\hline & DMP & $\hat{y}=1,5782+0,0078 x$ & $0,30^{\text {ns }}$ \\
\hline \multirow{3}{*}{ L4P4 } & Macro & $\hat{y}=0,0929+0,0026 x$ & $0,71^{* *}$ \\
\hline & Micro & $\hat{y}=0,3387+0,0003 x$ & $0,0085^{\mathrm{ns}}$ \\
\hline & DMP & $\hat{y}=1,2865+0,0205 x$ & $0,56 *$ \\
\hline \multirow{3}{*}{ LCPD } & Macro & $\hat{y}=0,1013+0,0016 x$ & $0,56^{*}$ \\
\hline & Micro & $\hat{y}=0,3368-6 E-05 x$ & $0,003^{n s}$ \\
\hline & DMP & $\hat{y}=0,7304+0,0231 x$ & $0,42^{\text {ns }}$ \\
\hline
\end{tabular}

CERR: solo com vegetação natural; PCCA = solo com pastagem contínua e adubada; L IP3: solo com lavoura de soja de um ano e três anos com pastagem de Brachiaria brizantha; L4P4: solo com lavoura de quatro anos e quatro anos com pastagem de Panicum maximum; LCPD: solo com lavoura de soja em sistema de plantio direto. ${ }^{\text {ns }}=$ não significativo, $*$ e $* *$ significativo a 5 e $1 \%$, respectivamente. sistema de manejo adotado (Quadro 4), confirmam a afirmação de Reichert et al. (2005), dequea infiltração de água no solo é um bom indicador dos efeitos da compactação do solo em função dos sistemas de manejo. Para os tratamentos PCCA e L4P 4 a correlação foi positiva, confirmandoa boa estruturação do sol o neste sistema.

A correlação entrea velocidadedeinfiltraçãobásica (VIB) e o diấmetro médio ponderado dos agregados (DMP) do solo em diferentes sistemas de manejo do solo no Cerrado encontram-se no quadro 4. Os tratamentos PCCA e L 4P 4 foram os queapresentaram relação positiva da VIB com o diâmetro médio geométrico, indicando que as pastagens contribuem para a agregação do solo. Deve-se ressaltar que no tratamento L1P3, onde ocorreu pastagem a cada três anos, a relação não significativa da VIB com o DMP poderia ser explicada pelo fato de que os três anos de pastagem nãoteriam sido suficientes para aumentar o diâmetro médio de maneira significativa. A participação relativa da infiltração acumulada do L 1P3, quefoi baixa entre os tratamentos (Quadro5), poderia corroborar essa ideia. NoCERR também não ocorreu relaçãosignificativa da VIB com o DMP, fato atribuído ao sistema radicular das espécies arbóreas, profundo, que poderia estar influenciando a infiltração deágua, independentemente das condições físicas do solo. Os altos valores da VIB e da infiltração acumulada do tratamento CERR reforçam a tese de que no solo natural, além das condiçốes físicas, o sistema radicular das plantas contribui para o processo de infiltração deágua nosolo.

\section{Infiltração acumulada de água no solo}

Os val ores médios deinfiltraçãoacumulada deágua nosol o dos diferentes sistemas demanejo sãomostrados noquadro5. Nãohouve efeitosignificativo da interação entre tratamento e ano ( $p>0,01)$, porém verificou-se efeitosignificativo dos tratamentos $(p \varangle 0,01)$.

Os maiores val ores de infiltraçãoacumulada foram verificados no solo sob vegetação natural de Cerrado. Considerando os valores rel ativos, entre os sistemas de manejo com interferência antropogênica, o PCCA foi o que apresentou a maior taxa de infiltração, especialmenteapós 2 h deteste. Todavia, ainda assim, seus resultados sempre foram menores que os do CERR. O maior valor do PCCA foi apenas $59,5 \%$ do CERR (0,5 h deteste).

O sistema de manejo L 1P3, a partir do tempo de $1 \mathrm{~h}$ de teste, e oLCPD, a partir de $3 \mathrm{~h}$, foram os que apresentaram a menor infiltração acumulada. No L1P3, essa redução é atribuída ao pisoteio animal, confirmando os dados obtidos por Lanzanova et al. (2005). No LCPD essa redução deve-se ao efeito do tráfego de máquinas eimplementos na compactação do solo. Esses efeitos são discuti dos nos trabal hos de Derpsch et al . (1997) eStone \& Silveira (2001).

Nos tratamentos PCCA e L4P4, embora apresentando valores médios de VIB de, 
Quadro 5. Infiltração acumulada de água no solo de diferentes sistemas de manejo do solo no Cerrado (média de sete anos) e a partici pação relativa de cada sistema em relação ao Cerrado

\begin{tabular}{|c|c|c|c|c|c|c|c|c|c|}
\hline \multirow{2}{*}{ Tempo } & \multicolumn{5}{|c|}{ Infiltração acumulada } & \multicolumn{4}{|c|}{ Participação relativa } \\
\hline & PCCA & LCPD & L4P4 & L1P3 & CERR & PCCA & LCPD & L4P4 & L1P3 \\
\hline $\mathrm{h}$ & & 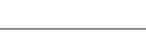 & $-\mathrm{mm}-$ & 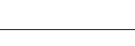 & - & 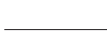 & - & & \\
\hline 0,5 & $56,86 \mathrm{~b}$ & $35,80 \mathrm{~b}$ & $49,64 \mathrm{~b}$ & $29,09 \mathrm{~b}$ & $95,60 \mathrm{a}$ & 59,48 & 37,45 & 51,93 & 30,43 \\
\hline 1,0 & $110,02 \mathrm{~b}$ & 69,02 b & $95,09 \mathrm{~b}$ & $55,00 \mathrm{c}$ & 189,95 a & 57,79 & 36,34 & 50,06 & 28,96 \\
\hline 2,0 & $207,34 \mathrm{~b}$ & $131,40 \mathrm{~cd}$ & $176,20 \mathrm{bc}$ & $101,43 \mathrm{~d}$ & $374,23 a$ & 55,40 & 35,11 & 47,08 & 27,10 \\
\hline 3,0 & $295,34 \mathrm{~b}$ & $182,70 \mathrm{c}$ & $249,84 \mathrm{~b}$ & $143,11 \mathrm{c}$ & $554,53 a$ & 53,26 & 32,95 & 45,05 & 25,81 \\
\hline 4,0 & $376,30 \mathrm{~b}$ & $230,00 \mathrm{c}$ & $316,18 \mathrm{~b}$ & $179,73 \mathrm{c}$ & 723,66 a & 52,00 & 31,78 & 43,69 & 24,84 \\
\hline 5,0 & $451,70 \mathrm{~b}$ & $274,70 \mathrm{~cd}$ & 376,17 c & $213,05 d$ & 911,47 a & 49,56 & 30,14 & 41,27 & 23,37 \\
\hline 6,0 & $520,84 \mathrm{~b}$ & $316,87 d$ & $432,64 \mathrm{c}$ & $244,02 d$ & $1089,10 \mathrm{a}$ & 47,82 & 29,10 & 39,73 & 22,41 \\
\hline
\end{tabular}

CERR: solo com vegetação natural; PCCA: solo com pastagem contínua e adubada; LIP3: solo com lavoura de soja de um ano e três anos com pastagem de Brachiaria brizantha; L4P 4: solo com lavoura de quatro anos e quatro anos com pastagem de Panicum maximum; e LCPD: solo com lavoura de soja em sistema de plantio direto. Letras iguais na linha (dentro de cada hora) não diferem entre si pelo teste de Tukey a $5 \%$.

respectivamente, 33,72 e $27,56 \mathrm{~cm} \mathrm{~h}^{-1}$, a infiltração acumulada foi maior para otratamento PCCA a partir de 5 h deteste. Assim, o manejo desolocom pastagem deBrachiaria decumbens deforma contínua apresenta menor risco ao escoamento superficial em relaçãoaos demais sistemas de manejo do solo, com exceção do tratamentoCERR.

\section{CONCLUSÕES}

1. O uso do sol o com sistema de lavoura contínua, pecuária contínua e integração lavoura-pecuária tem a velocidade de infiltração básica e infiltração acumulada reduzida em rel ação ao sol o com vegetação natural na região do Cerrado.

2. O uso do solo com pastagens causa menos impactos na capacidade deste em infiltrar água e na infiltração acumulada, quando comparado a sistema de integraçãolavoura-pecuária elavoura contínua.

3. Brachiaria brizantha cv. Marandu, quando introduzida em sistema direto após soja, não tem a mesma capacidade de manter a qualidade física do sol o em relação a Brachi aria decumbens cv. Basilisk com adubação de manutenção ePani cum maxi mum cv. Tanzânia introduzida após quatro anos de soja.

\section{LITE RATURA CITADA}

BERTOL, I.; GOMES, K.E.; DENARDIN, R.B.N.; ZAGO, L.A. \& MARASCHIN, G.E. Propriedades físicas do solorelacionadas a diferentes níveis de oferta de forragem numa pastagem nativa. Pesq. Agropec. Bras., 35:25-38, 1997.
BERTOL, I.; BEUTLER, J .F.; LEITE, D. \& BATISTELA, O. Propriedades físicas de um Cambissolo Húmico afetadas pelo tipo de manejo do solo. Sci. Agríc., 58:555-560, 2001.

BONO, J .A.M. \& MACEDO, M.C.M. Sistemas de recuperação de pastagens degradadas sob um Latossolo Roxo e alterações na taxa de infiltração de. In: CONGRESSO LATINO AMERICANO DE CIÊNCIA DO SOLO, 13., Águas de Lindoia, 1996. Anais... Águas de Lindoia, SBCS/ USP/SLCS/CEA/SBM, 1996. CD-ROM.

BONO, J.A.M.; MACEDO, M.C.M.; \& EUCLIDES, V.P.B. Propriedades físicas de um Latossolo Vermelho Escuro, sob pastagem, alterações em função do manejo. In: CONGRESSO BRASILEIRO DE CIÊNCIA DO SOLO, 25, 1995, Viçosa, Anais ... Viçosa: SBCS/UFV, 1995. p.1893-1895.

BRANDÃO, V.S.; PRUSKI, F.F. \& SILVA, D.D. Infiltração da água no solo. Viçosa, MG, Universidade F ederal de Viçosa, 2003. 98 p.

CHEN, C. \& WAGENET, R.J. Simulations of water and chemicals in macropore soil. Part 1 . Representation of the equivalent macrospore influence and its affect on soil water flow. J. Hydrol., 130:105-126, 1992

DERPSCH, R.; SIDIRAS, N. \& HEINZMANN, F.X. Manejo do solo com coberturas verdes de inverno. Pesq. Agropec. Bras., 20:761-773, 1997.

EMPRESA BRASILEIRA DE PESQUISA AGROPECUÁRIA EMBRAPA. Serviço Nacional de Levantamento e Conservação de Solo. Manual de métodos de análise de solo. Rio de J aneiro, 1997 . 212p.

FABIAN, A.J . \& OTTONI FILHO, T.B. Determinação de curvas de infiltração usando câmara de fluxo. R. Bras. Ci. Solo, 21:325-333, 1997.

FERRI, M.G. Ecologia dos Cerrados. In: FERRI, M.G., coord. SIMPÓSIO SOBRE O CERRADO, Bases para utilização agropecuária. São Paulo, USP/Itatiaia, 1977. p.15-33. 
FORSYTHE, W. Física de suelos: Manual de laboratório. San J osè, IICA, 1985. 212p.

FREITAS J ÚNIOR, E. \& SILVA, E.M. Uso da centrífuga para determinação da curva de retenção de água no solo, em uma única operação. Pesq. Agropec. Bras., 19:1423-1428, 1984.

FREITAS J ÚNIOR, E. \& LUCHIARI JÚNIOR, A. Manejo: Aspectos físicos. In: PEREIRA, V.P.; FERREIRA, M.E. \& CRUZ, M.C.P., eds. Solos altamente suscetíveis à erosão. J aboticabal, FCAV-UNESP/SBCS, 1994. 253p.

GUEDES, H.M.; RESCK, M.V.S.; PERREIRA, I.S.; SILVA, J .E.; CASTRO, L.H.R. Caracterização da distribuição do tamanho de agregados de diferentes sistemas de manejo e seu conteúdo de carbono em Latossolo Vermel ho-Escuro na região dos cerrados, Brasil In: PERREIRA, R.C. \& NASSER, L.C.B. eds. SIMPÓSIO SOBRE O CERRADO, 7, 1996, INTERNATIONAL SYMPOSIUM ON TROPICAL SAVANNAS, 1, BRASÍLIA, 1996. Anais... Planaltina: EMBRAPA-CPAC. 1996. p.329-333.

KANNO, T.; MACEDO, M.C.M.; UOZUMI, S.; EUCLIDES, V.P.B.; BONO, J .A.M.; YOSHIMURA, Y.; CORREA, M.R. \& SANTOS J r., J.D.G. Development of grassland management Technology for sustainable agropastoral systems in the sub-tropical zone in Brazil. In: KANNO, T. $\&$ MACEDO, M.C.M., eds. WORKSHOP ON AGROPASTORAL SYSTEM IN SOUTH AMERICA, Campo Grande, 2001. Proceedings... Campo Grande, J IRCAS/E mbrapa Gado de Corte, 2001. p.63-73.

KANNO, T.; MACEDO, M.C.; EUCLIDES, V.P.B.; BONO, J .A.; SANTOS, J.D.G.; ROCHA, M.C. \& BERETTA, L.G.R. Root biomass of five tropical grass pastures under continuous grazing in brazilian savannas. Grassland Sci., 45:9-14, 1999.

KEMPER, W.D. \& CHEPIL W.S. Size distribution of aggregates. In: BLACK, C.A., ed. Methods of soil analysis. Madison, American Society of Agronomy, 1965. p.499510.

KLUTHCOUSKI, J . \& STONE, L.F. Manejo sustentável dos solos dos Cerrados. In: KLUTHCOUSKI, J .; STONE, L.F. \& AIDAR, H., eds. Integração lavoura-pecuária. Goiânia, Embrapa Arroz e Feijão, 2003. p.61-104.

LAL, R. Soil erosion from tropical arable lands and its control. Adv. Agron., 37:183-247, 1984.

LANZANOVA, M.E.; LOVATO, T.; NICOLOSO, R.S.; GIRADELLO, V.; SILVA, V.L. \& BRAGAGNOLO, J. Efeito do pisoteio bovino na infiltração de água de um Argissolo Vermelho-Amarelo. In: CONGRESSO BRASILEIRO DE CIÊNCIA DO SOLO, 30., Recife, 2005. Anais... Recife, Sociedade Brasileira de Ciência do Solo, 2005. CD-ROM.

PAGOTTO, D.S. Comportamento do sistema radicular, reservas orgânicas e mineralização líquida do nitrogênio do sol o em pastagem irrigada de capim Tanzânia submetida a diferentes intensidades de pastejo. Piracicaba, Escola Superior Agricultura Luiz de Queiroz, 2001. (Tese de Mestrado).
PIMENTEL-GOMES, F. \& GARCIA, C.H. Estatística aplicada a experimentos agronômicos e florestais. Piracicaba, Escola Superior Agricultura Luiz de Queiroz, 2002. 309p.

PRUSKI, F.F.; VENDRAME, V.; OLIVEIRA, E.F.; BALBINO, L.C.; FERREIRA, P.A.; WERLANG, L. \& CARVALHO, L.T. Infiltração da água num Latossolo Roxo. Pesq. Agropec. Bras., 32:77-84, 1997.

REICHARDT, K. \& TIMM, L.C. Solo, planta e atmosfera: Conceitos, processos e aplicações. Barueri, Manole, 2004. 478p.

REICHERT, J.M.; REINERT, D.J .; CASSOL, E.A. \& SILVA, V.R. A infiltração da água no solo sob manejo conservacionista. In: CONGRESSO BRASILEIRO DE CIÊNCIA DO SOLO, 30., Recife, 2005. Anais... Recife, Sociedade Brasileira de Ciência do Solo, 2005. CD-ROM.

RESENDE, M.; KER, J.C. \& BAHIA, A.F.C. Desenvolvimento sustentado do cerrado. In: ALVAREZ. V.H. \& FONTES, L.E.; FONTES, M.P.F. eds. O solo nos grandes domínio morfoclimáticos do Brasil e o desenvol vimento sustentado, Viçosa, SBCS/UFV, 1996. p.169-199.

SALTON, J.C.; HERNANI, L.C.; BROCH, D.L. \& FABRÍCIO, A.C. Alterações em atributos físicos do solo decorrentes da rotação soja-pastagem, no sistema plantio direto. Dourados, Embrapa Agropecuária Oeste, 1999. 5p. (Comunicado Técnico, 10)

SILVA, C.L. \& KATO, E. Avaliação de modelos para previsão da infiltração de água em solos sob Cerrados. Pesq. Agropec. Bras., 33:1149-1158, 1998.

SILVA, C.L. \& KATO, E. Efeito do selamento superficial na condutividade saturada da superfície de um solo sob Cerrado. Pesq. Agropec. Bras., 32:213-220, 1997.

SILVA, M.L.N.; BLANCANEAUX, P.; CURI, N.; LIMA, J .M.; MARQUES, J.J.G.S. \& CARVALHO, A.M. Estabilidade e resistência de agregados de Latossolo Vermelho-Escuro cultivado com sucessão mil ho-adubo verde. Pesq. Agropec. Bras., 33:97-103, 1998.

STONE, L.F. \& SILVEIRA, P.M. Efeitos do sistema de preparo e da rotação de culturas na porosidade e densidade do solo. R. Bras. Ci. Solo, 25:395-401, 2001.

VEZZANI, F.M. Qualidade do sistema solo na produção agrícola. Porto Alegre, Universidade Federal Rio Grande do Sul, 2001. 184p. (Tese de Doutorado)

VILELA, L. MACEDO, M.C.M.; MARTHA JÚNIOR, G.B. \& KLUTHCOUSKI, J. Degradação de pastagens e indicadores de sustentabilidade, In: KLUTHCOUSKI, J .; STONE, L.F. \& AIDAR, H. eds. Integração Lavourapecuária, Goiânia: Embrapa- Arroz e Feijão, 2003. p.107127.

ZIMMER, A.H.; MACEDO, M.C.M.; BARCELLOS, A.O. \& KICHEL, A.N. Estabelecimento e recuperação de pastagem de Brachiaria. In: SIMPÓSIO SOBRE MANEJ O DE PASTAGEM, 11., Piracicaba, 1994. Anais... Piracicaba, Escola Superior Agricultura Luiz de Queiroz, 1994. p.153-208. 